\title{
High-brightness electron beams for linac-based bunched beam electron cooling
}

D. Kayran $\oplus^{*}$, Z. Altinbas, D. Bruno, M. R. Costanzo, A. Drees, A. V. Fedotov, W. Fischer, M. Gaowei, D. M. Gassner, X. Gu, R. Hulsart, P. Inacker, J. Jamilkowski, Y. Jing, P. Kankiya, J. Kewisch, C. Liu, J. Ma, K. Mernick, T. A. Miller, M. Minty, L. K. Nguyen, M. Paniccia, I. Pinayev, V. Ptitsyn, V. Schoefer, S. Seletskiy, F. Severino, T. C. Shrey, L. Smart, K. S. Smith, A. Sukhanov, P. Thieberger, J. Tuozzolo, E. Wang, G. Wang, W. Xu, A. Zaltsman, He Zhao, and Zhi Zhao Brookhaven National Laboratory, Upton, New York 11973, USA

(Received 22 November 2019; accepted 23 January 2020; published 21 February 2020)

A high-current high-brightness electron accelerator for low-energy RHIC electron cooling (LEReC) was successfully commissioned at Brookhaven National Laboratory. The LEReC accelerator includes a dc photoemission gun, a laser system, a photocathode delivery system, magnets, beam diagnostics, a superconducting rf booster cavity, and a set of normal conducting rf cavities to provide enough flexibility to tune the beam in the longitudinal phase space. Cooling with nonmagnetized if accelerated electron beams requires longitudinal corrections to obtain a small momentum spread while preserving the transverse emittances. Electron beams with kinetic energies of 1.6 and $2.0 \mathrm{MeV}$ with a beam quality suitable for cooling were successfully propagated through $100 \mathrm{~m}$ of beam lines, including dispersion sections, maintained through both cooling sections in RHIC and used for cooling ions in both RHIC rings simultaneously. The beam quality suitable for cooling RHIC beams was achieved in 2018, which led to the first experimental demonstration of bunched beam electron cooling of hadron beams in 2019 .

DOI: 10.1103/PhysRevAccelBeams.23.021003

\section{INTRODUCTION}

A new, state-of-the-art, electron accelerator for cooling of low-energy RHIC ion beams (LEReC) was built and commissioned at Brookhaven National Laboratory (BNL) [1]. The purpose of LEReC is to increase the luminosity in RHIC operation at low collision energies. Collisions at these low energies are part of the RHIC beam energy scan phase II (BES-II), a search for a critical point in the quantum chromodynamics (QCD) phase diagram [2-3].

Electron cooling is a well-established method to increase phase-space density of the heavy particles by their interaction with copropagated electrons. The first electron cooling was proposed by Budker in 1966 and demonstrated in 1974 [4]. Electron cooling is successfully used at different facilities around the world [5]. The highest-energy electron cooling using a dc electron beam was demonstrated at Fermi National Accelerator Laboratory (FNAL) [6].

\footnotetext{
*dkayran@bnl.gov

Published by the American Physical Society under the terms of the Creative Commons Attribution 4.0 International license. Further distribution of this work must maintain attribution to the author(s) and the published article's title, journal citation, and DOI.
}

Unlike all other electron coolers to date, LEReC uses if accelerated bunched electron beams [7]. Such a cooling scheme with a bunched electron beam is one of the most promising approaches for high-energy electron cooling, since bunches can be accelerated using rf cavities to the required high energies. For cooling, the electron beam must have not only sufficiently low transverse emittances but, more importantly, a small energy spread. This is particularly challenging for $\mathrm{rf}$ accelerated beams.

The layout of LEReC is shown in Fig. 1. The LEReC accelerator includes a photocathode dc gun with a high-power laser system, magnets, beam diagnostics, a superconducting rf (SRF) booster cavity, and a set of normal conducting rf cavities to provide enough flexibility to tune the beam in the longitudinal phase space. LEReC is designed to provide electron beams for cooling RHIC gold ions at energies between 3.85 and $5.75 \mathrm{GeV}$ per nucleon.

LEReC uses a dc photocathode gun built by Cornell University [8]. The gun tests with beam started in 2017 when the gun delivered up to $10 \mathrm{~mA}$ average current [9]. Electron bunches are generated by illuminating a multialkali $\left(\mathrm{CsK}_{2} \mathrm{Sb}\right.$ or $\left.\mathrm{NaK}_{2} \mathrm{Sb}\right)$ photocathode [10] with a green high-power fiber laser (wavelength of $520 \mathrm{~nm}$ ) [11] equipped with sophisticated laser transport and stabilization systems $[12,13]$. 


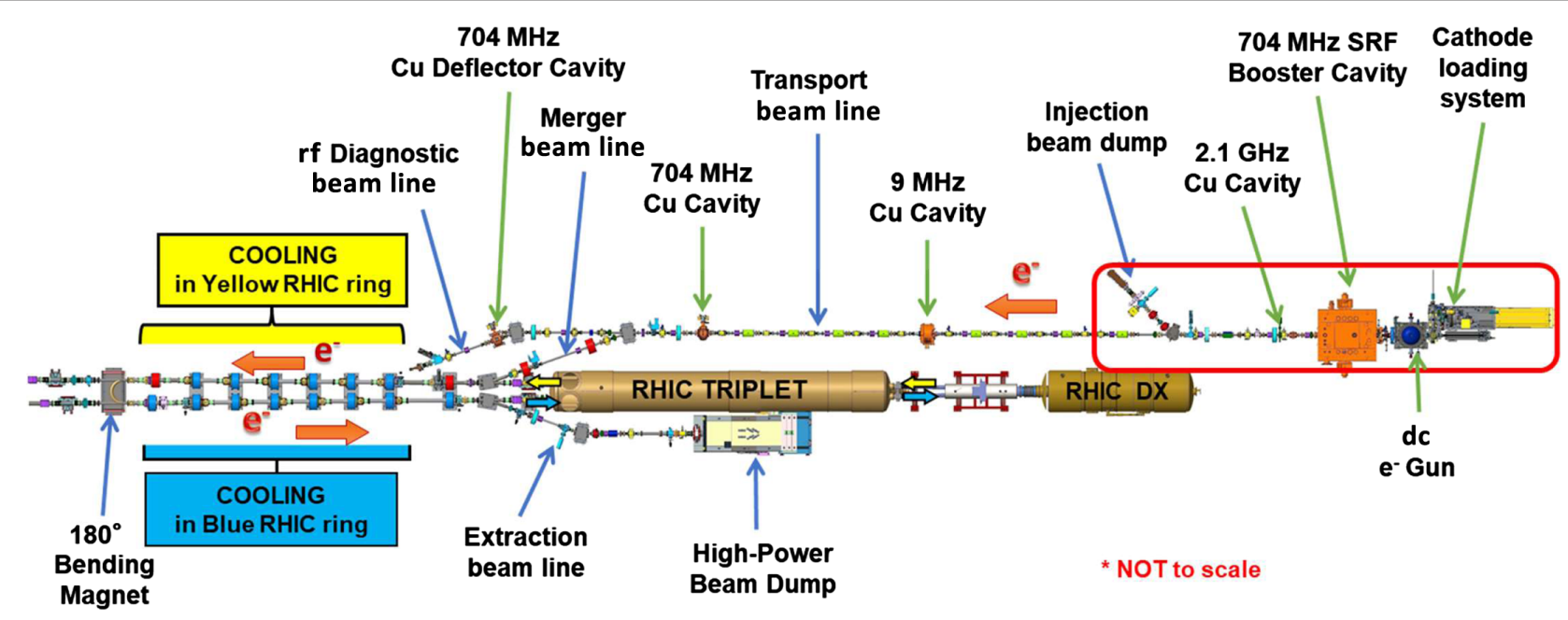

FIG. 1. Layout of the LEReC accelerator. The red contour box indicates the dc gun test area.

To minimize the cathode exchange time, three multicathode carriers were built. Each cathode carrier can hold up to 12 photocathodes. A carrier is attached to the gun with a vacuum pressure on the $10^{-11}$ Torr range [14]. For the initial LEReC gun tests with a beam in 2017-2018, we used a large active cathode area with a $12 \mathrm{~mm}$ diameter. Later, in order to reduce the cathode quantum efficiency (QE) degradation due to the ion back bombardment in high current operation, $6 \mathrm{~mm}$ diameter active area cathodes shifted by $4 \mathrm{~mm}$ off center have been used (see Fig. 2).

The $350-400 \mathrm{keV}$ electron beam from the gun is transported to a $704 \mathrm{MHz}$ SRF booster cavity, where electron bunches are accelerated off crest to produce an energy chirp, and to a $2.1 \mathrm{GHz}$ third-harmonic normal conductive "linearizing" cavity. The electron beam can be accelerated to a maximum kinetic energy of $2.6 \mathrm{MeV}$. Downstream of the third-harmonic cavity, the electron bunch is ballistically stretched in the 30-m-long transport beam line to the required bunch length. The energy chirp is compensated by a normal conductive $704 \mathrm{MHz}$ cavity before entering the first cooling section. A $9 \mathrm{MHz}$ normal conducting rf cavity is used to remove bunch-to-bunch energy variations along

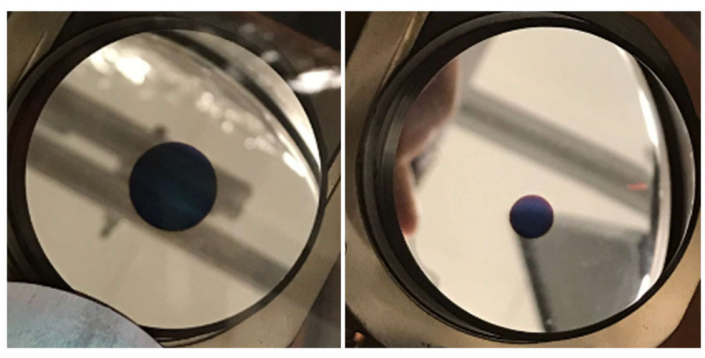

FIG. 2. LEReC cathode pucks with deposited cathode material. Left: Active cathode area of $12 \mathrm{~mm}$ diameter in the center used during the gun commissioning in 2017. Right: Active cathode area of $6 \mathrm{~mm}$ diameter shifted by $4 \mathrm{~mm}$ from the center used during LEReC high-current operation in 2018 and 2019. one macrobunch caused by beam loading in the rf cavities. The electron beam is merged into RHIC to cool the ion beam in the RHIC yellow ring first. Then the electron beam is transported through the $180^{\circ}$ bend for cooling ions in the blue RHIC ring. Finally, the electrons are extracted from the RHIC cooling loop and sent through the extraction beam line to the high-power (HP) beam dump. Simulation results of longitudinal gymnastic from the cathode to the HP beam dump for a bunch with charge of $75 \mathrm{pC}$ and kinetic energy of $1.6 \mathrm{MeV}$ are shown in Fig. 3.

Design and commissioning of the rf cavities are described in Refs. [15-20]. The optics of the entire transport line has been designed and optimized to deliver electron bunches of different energies with an electron beam quality suitable for cooling [21]. The LEReC design beam parameters for cooling at the lowest BES-II energies are summarized in Table I. Only two of the lowest energies were commissioned as requested by the physics program during the run of 2019.

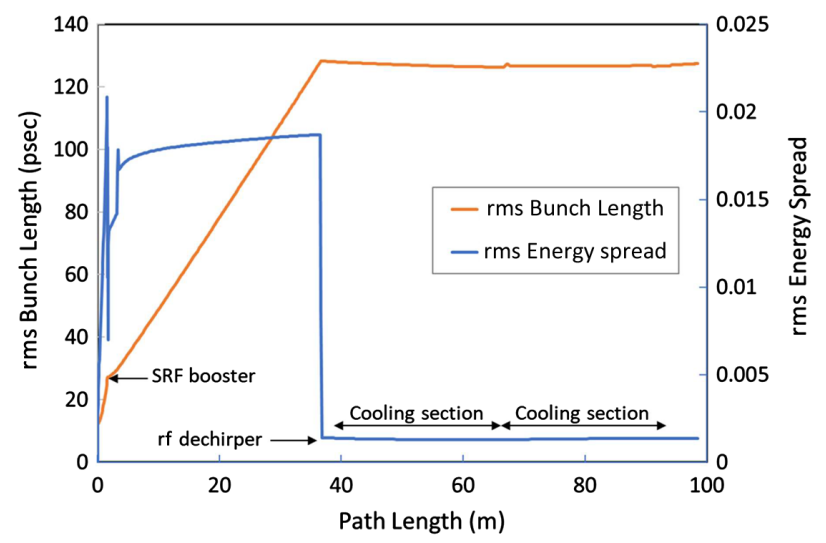

FIG. 3. Simulated rms bunch length and rms energy spread along the beam line from the gun to the beam dump for a bunch charge of $75 \mathrm{pC}$ and a kinetic energy of $1.6 \mathrm{MeV}$. 
TABLE I. Electron beam baseline parameters.

\begin{tabular}{lccc}
\hline \hline Kinetic energy, MeV & 1.6 & 2.0 & 2.6 \\
Bunch charge, pC & 130 & 160 & 200 \\
Bunches per train & 30 & 27 & 24 \\
Macrobunch charge, nC & 3.9 & 4.3 & 4.8 \\
Macro bunch rep. f, MHz & 9.1 & 9.1 & 9.1 \\
Total beam current, mA & 36 & 40 & 45 \\
Normalized emittance, $\mu \mathrm{m}$ & $<2.5$ & $<2.5$ & $<2.5$ \\
Energy spread, $10^{-4}$ & $<5$ & $<5$ & $<5$ \\
\hline \hline
\end{tabular}

The LEReC laser system is designed to operate in several modes: the $1 \mathrm{~Hz}$ pulsed mode, the $76 \mathrm{kHz}$ mode, and the $9 \mathrm{MHz}$ continuous-wave (CW) mode. An ultrafast pulse picker technique [22] has been developed to generate the optical bunch time structure with $9.1 \mathrm{MHz}$ macrobunches consisting of 30 pulses of $704 \mathrm{MHz}$ frequency. In the $1 \mathrm{~Hz}$ pulsed mode, from one to several thousand optical macrobunches at a $1 \mathrm{~Hz}$ rate are picked up to produce a corresponding number of electron macrobunches.

In the $76 \mathrm{kHz}$ mode, the same system allows one to create a train of 1-6 optical macrobunches at a $76 \mathrm{kHz}$ rate. The number of macrobunches per train in the $76 \mathrm{kHz}$ mode is limited by the $5 \%$ duty cycle of the Pockels cell utilized in the pulse picking. The $76 \mathrm{kHz}$ mode is important for initial RHIC cooling commissioning and optimization. Because of the moderate average laser power as well as the moderate average electron beam current, no electron beam quality degradation is expected in this mode in comparison to the pulsed mode. In the $76 \mathrm{kHz}$ mode, the train repetition frequency is matched with the RHIC revolution frequency at the RHIC operational energy. In this case, the selected RHIC ion bunches interact with the electron macrobunches every time the ion bunches go through the LEReC cooling section as illustrated in Fig. 4.

In the $9 \mathrm{MHz} \mathrm{CW}$ mode, all $9.1 \mathrm{MHz}$ optical macrobunches are used to generate high-current electron beams. The repetition rate of optical macrobunches is set to be the same as the repetition rate of the RHIC ion bunches. As a result, each ion bunch interacts with the electrons in the cooling section.

\section{BEAM QUALITY MEASUREMENTS AND OPTIMIZATION}

The commissioning of the full accelerator started in March of 2018. The initial commissioning was carried out in the pulsed beam mode. The bunch quality measurement and optimization were performed in the $1 \mathrm{~Hz}$ pulsed mode. The LEReC machine protection system (MPS) was fully operational during commissioning. The details of the LEReC beam instrumentation and the MPS were reported in Refs. $[23,24]$, respectively. Below, we describe the beam quality measurements performed during the accelerator commissioning which were the most critical to demonstrate electron cooling. Results of beam quality optimization presented in the next sections are for the bunch charge of $75 \mathrm{pC}$, which was found the optimum bunch charge during cooling studies.

\section{A. Longitudinal beam quality}

The electron beam rms energy spread in the cooling sections is required to be less than $5 \times 10^{-4}$, which corresponds to an absolute energy spread better than $1 \mathrm{keV}$ at the LEReC operation energies of $1.6-2 \mathrm{MeV}$. At first, special care was taken to make sure that there were no gun high-voltage power supply ripples exceeding $1 \mathrm{kV}$ [25]. To avoid energy spread buildup in the cooling sections due to the longitudinal space-charge effect, the electron bunches are ballistically stretched up to $400 \mathrm{ps}$ in $30 \mathrm{~m}$ of the transport line. To measure and control the longitudinal distribution of the beam before injecting it in the first cooling section, a dedicated rf diagnostic line was built.

The schematic layout of the rf diagnostic line is shown in Fig. 5. It includes a solenoid lens to provide small beta functions at a yttrium-aluminum-garnet (YAG) screen location, a $20^{\circ}$ dipole to generate a dispersion of $0.8 \mathrm{~m}$,
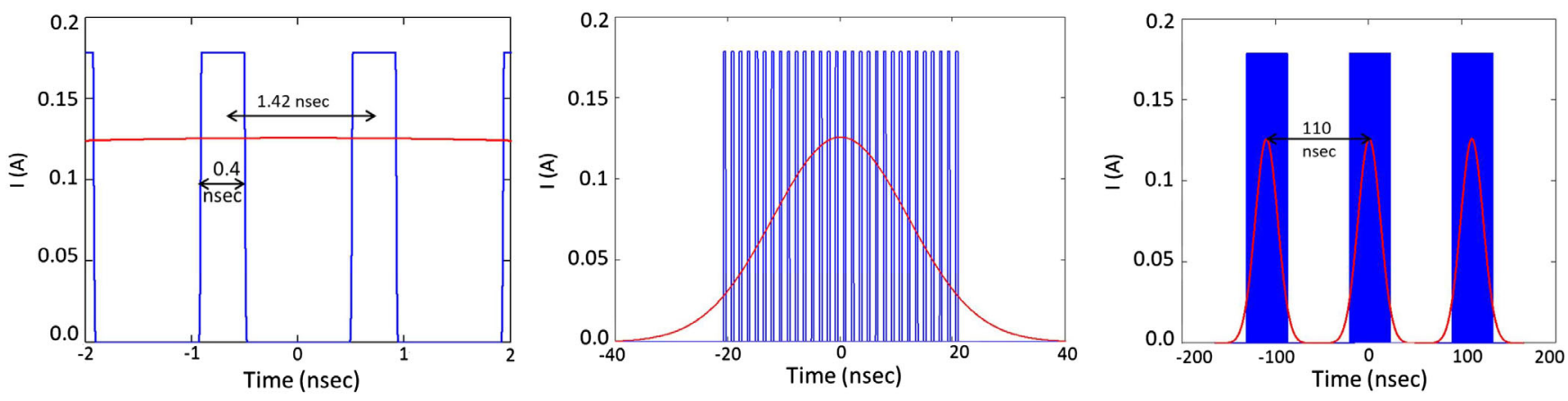

FIG. 4. LEReC and RHIC beam time structure in the cooling sections. The left shows two electron bunches spaced by 1.42 ns (corresponding to the $704 \mathrm{MHz}$ LEReC laser maximum repetition frequency). The center shows one LEReC macrobunch consisting of 30 electron bunches (blue) placed over a single ion bunch (red). The right shows a train of three electron macrobunches spaced by $110 \mathrm{~ns}$ (corresponding to the $9.1 \mathrm{MHz}$ RHIC repetition frequency) overlapped with the three ion bunches. 


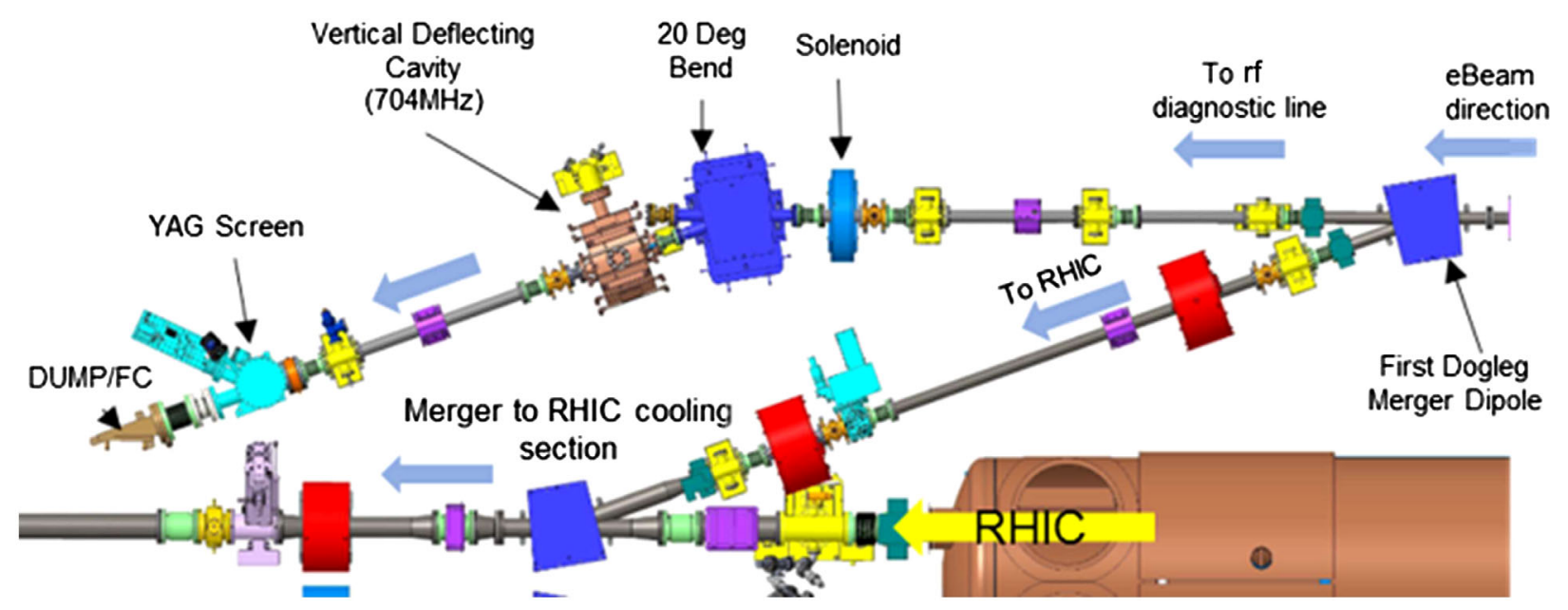

FIG. 5. The rf diagnostic line layout.

and a $704 \mathrm{MHz}$ deflecting cavity to provide vertical timedependent kicks. When the first dogleg merger dipole is turned off, the electron beam is transported to the $\mathrm{rf}$ diagnostic line to measure the longitudinal phase-space distribution.

The longitudinal phase-space optimization is conducted by tuning the voltages and phases of the rf cavities while observing the bunch profile on the YAG screen. An example of a longitudinal phase-space measurement after
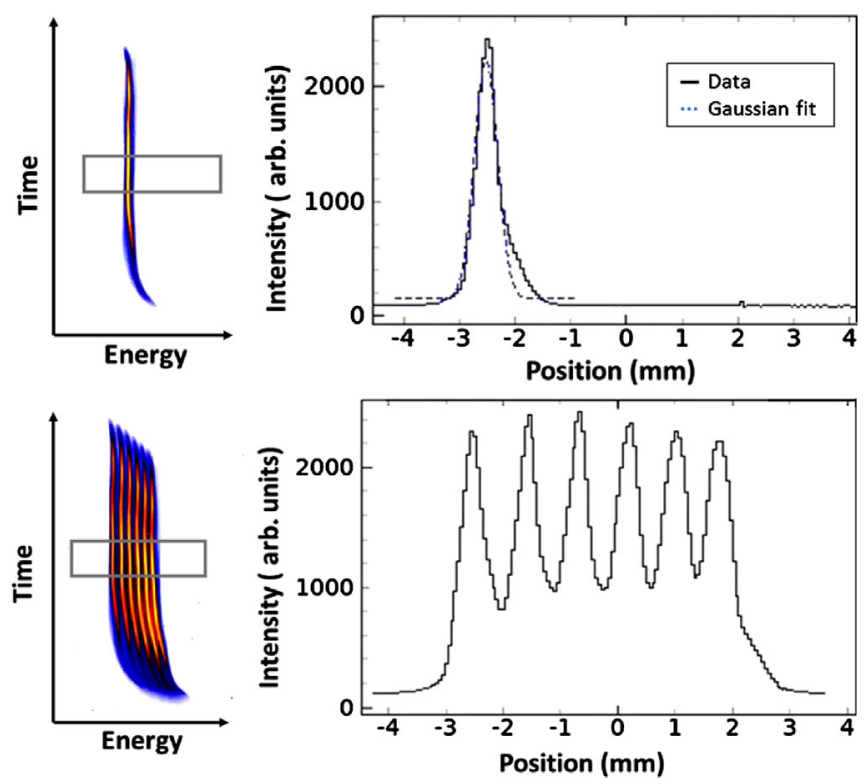

FIG. 6. Longitudinal phase-space measurements at the rf diagnostic line with a transverse deflecting cavity and a YAG profile monitor for bunch charges of $75 \mathrm{pC}$. The beam images (left) show at the top a single macrobunch and at the bottom a train of six macrobunches. The intensity profiles (right) are taken over the indicated windows and are used to determine the energy distribution, including differences along the six-macrobunch train. the optimization for a bunch charge of $75 \mathrm{pC}$ is shown in Figs. 6 and 7. The horizontal rms size of the macrobunch center slice is $0.16 \mathrm{~mm}$, which includes both the dispersionrelated and the emittance-related beam sizes. The conclusion from this measurement is that a relative energy spread of less than $2 \times 10^{-4}$ or an absolute energy spread of $400 \mathrm{eV}$ was achieved. By proper adjustment of the voltage and phase of the $9 \mathrm{MHz}$ cavity, the beam loading effect along a single macrobunch was well compensated. The energy difference between macrobunches during pulsed operation with several macrobunches was measured to be $0.69 \mathrm{keV} / \mathrm{nC}$. Later, during cooling commissioning and optimization, we used the energy differences between macrobunches for easier matching of the electron and ion beam Lorentz $\gamma$ factors in the RHIC cooling sections [26].

\section{B. Transverse beam quality}

Because of the low beam energy, the beam dynamics in the LEReC accelerator is dominated by space-charge effects. The transverse electron beam emittance in the

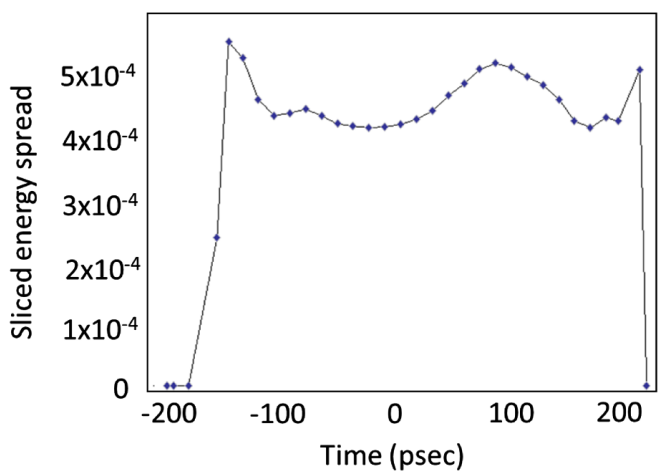

FIG. 7. Result of the longitudinal phase-space optimization. The sliced energy spread is measured at the rf diagnostic line YAG profile monitor for a bunch charge of $75 \mathrm{pC}$ with a FWHM of $400 \mathrm{ps}$. 

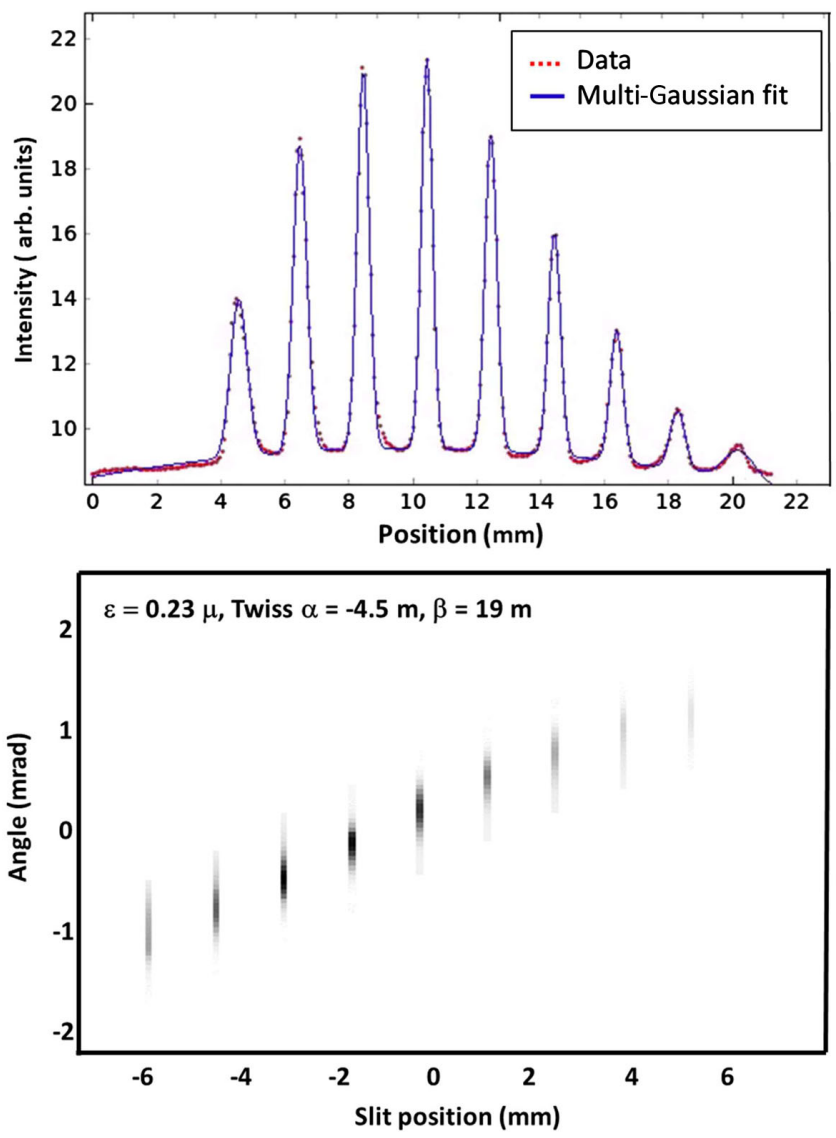

FIG. 8. Phase-space measurements in the injection line using a multislit mask for a bunch charge of $75 \mathrm{pC}$. Top: Vertical intensity profile at the YAG profile monitor when a multislit mask is inserted upstream. Bottom: Result of the transverse phase-space reconstruction at the multislit mask location with the listed geometric emittance $\varepsilon$ and the Twiss parameters $\alpha$ and $\beta$.

injection line was characterized with a multislit system (see Fig. 8) [27]. In the RHIC cooling sections, the emittance was measured using movable slits. Details about emittance measurements using the multislits can be found in Ref. [28].

The electron beam transverse phase space is matched to the RHIC beam phase space by adjusting the last transport line solenoid and the first solenoids in the cooling sections. Solenoid lenses installed every $3 \mathrm{~m}$ in both cooling sections are used to compensate the transverse electron beam spacecharge effects. The results of the optimized matching to the cooling sections for a bunch charge of $75 \mathrm{pC}$ are shown in Fig. 9. The normalized transverse rms emittances in both cooling sections are lower than $1.6 \mu \mathrm{m}$.

\section{HIGH-CURRENT OPERATION}

\section{A. Gun tests in CW operation}

The initial studies of the high-current gun operation were carried out using the gun test beam line indicated by the red box in Fig. 1. In April 2018, the first CW electron beam was transported through the rf cavities. An average current of $1.3 \mathrm{~mA}$ was delivered to the injection beam dump. The injection beam dump is designed to accept an average beam power of up to $14 \mathrm{~kW}$ at energies of up to $0.5 \mathrm{MeV}$. In order to proceed with high-current testing in the gun test line, the SRF booster voltage was reduced.

By September of 2018, we were able to deliver a stable $30 \mathrm{~mA}$ beam current to the injection beam dump using a reduced SRF booster voltage. This mode of operation was used to study gun stability and long-term cathode QE lifetime [25]. During several hours of high-current operation, we observed a small recovery of the cathode QE instead of a QE degradation, as shown in Fig. 10. The slowly recovering cathode QE was confirmed repeatedly during the 2019 run at different operational average currents from 1 to $30 \mathrm{~mA}$. The observed recovery of cathode QE is attributed to the cathode cleaning process as a result of high-current operation. A similar cathode $\mathrm{QE}$ improvement during operation at a beam current of $1 \mathrm{~mA}$ is reported in Ref. [29].

\section{B. CW operation in the final LEReC setup}

By the summer of 2018, the CW beam was transported to the high-power beam dump. We were able to deliver a beam average power of $32 \mathrm{~kW}$ to the HP beam dump for a few minutes. During the $\mathrm{CW}$ operation at a high current, we observed increased vacuum pressure in the HP beam region, presumably as a result of the beam halo scraping the dump entrance flange. After opening the HP beam dump at the end of the run 2018, we observed overheating marks at the entrance flange and at the cone tip of the HP beam dump (see Fig. 11).

As a result of these observations, the HP beam dump and the extraction beam line were redesigned for the following run. The entrance flange of the beam dump was replaced with a flange of larger aperture. The last quadrupole magnet in the beam dump line was replaced with an overfocusing solenoid in order to evenly spread the electron beam inside the beam dump. As a result of these modifications, during the run of 2019 we were able to transport a high-current $\mathrm{CW}$ beam with an average power of about $40 \mathrm{~kW}$ to the HP beam dump with acceptable vacuum and temperature growth (Fig. 12).

To allow more stable operation with a high-current beam, it was important to implement a beam intensity feedback system. The beam intensity feedback includes two stages. The signal from the current transformer installed downstream of the gun is used as a pickup signal to compensate any slow changes in the cathode QE by adjusting the half wave plate installed in the laser trailer. For laser power fluctuation compensation, the system stabilizes laser power delivered to the cathode. The signal from the photodiode, installed at the gun table in the tunnel, is used to control the 

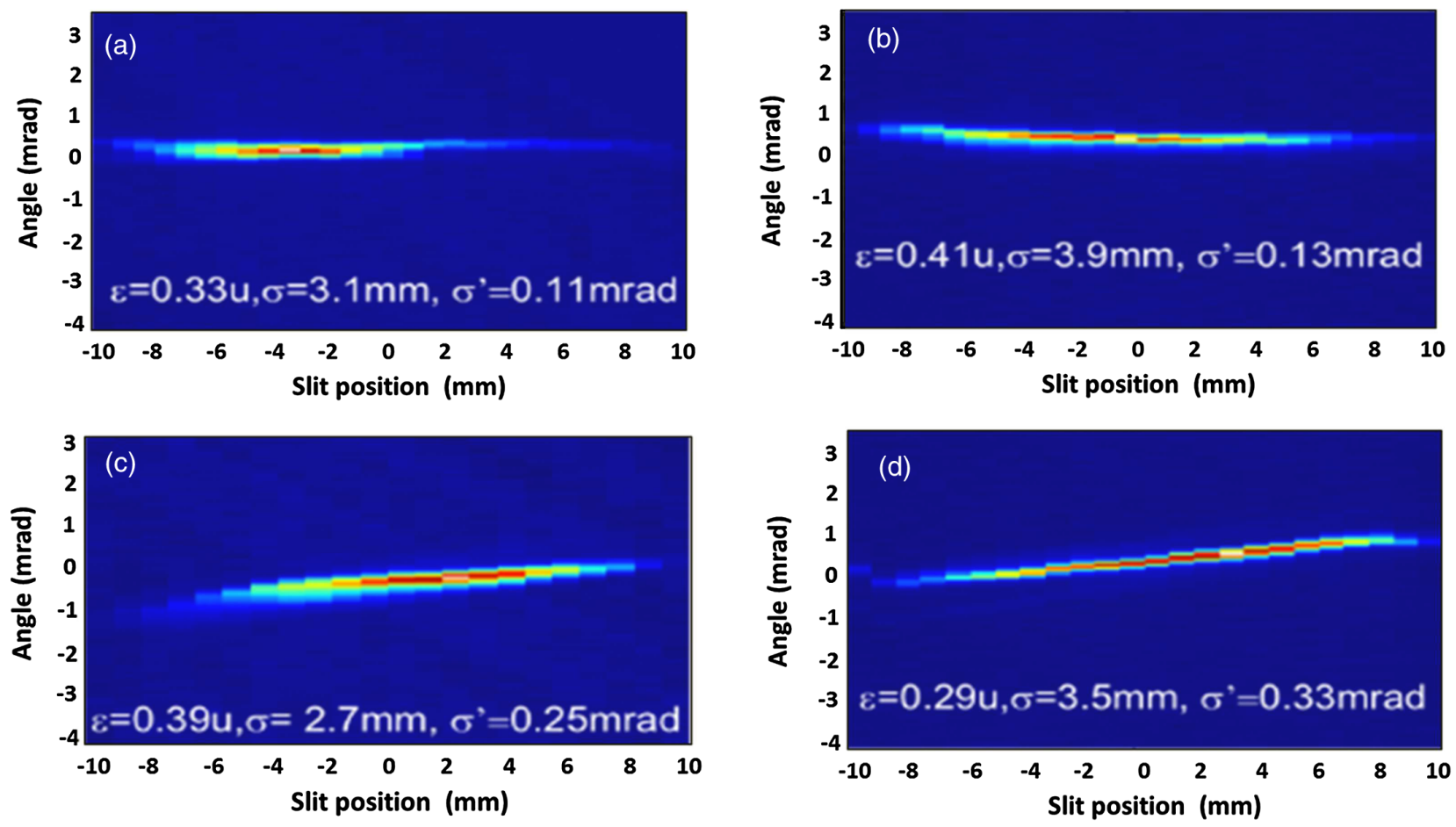

FIG. 9. Transverse phase-space measurements in the two cooling sections using movable slits: (a),(b) horizontal and vertical phase space in the yellow cooling section and (c),(d) horizontal and vertical phase space in the blue cooling section. Each plot lists the respective geometric emittance $(\varepsilon)$, the rms beam size $(\sigma)$, and the rms beam angular spread $\left(\sigma^{\prime}\right)$.

voltage of the acousto-optic modulator (AOM), installed in the laser trailer, to adjust the laser power. Results of beam current stabilization with the intensity feedback are shown in Figs. 10 and 12.
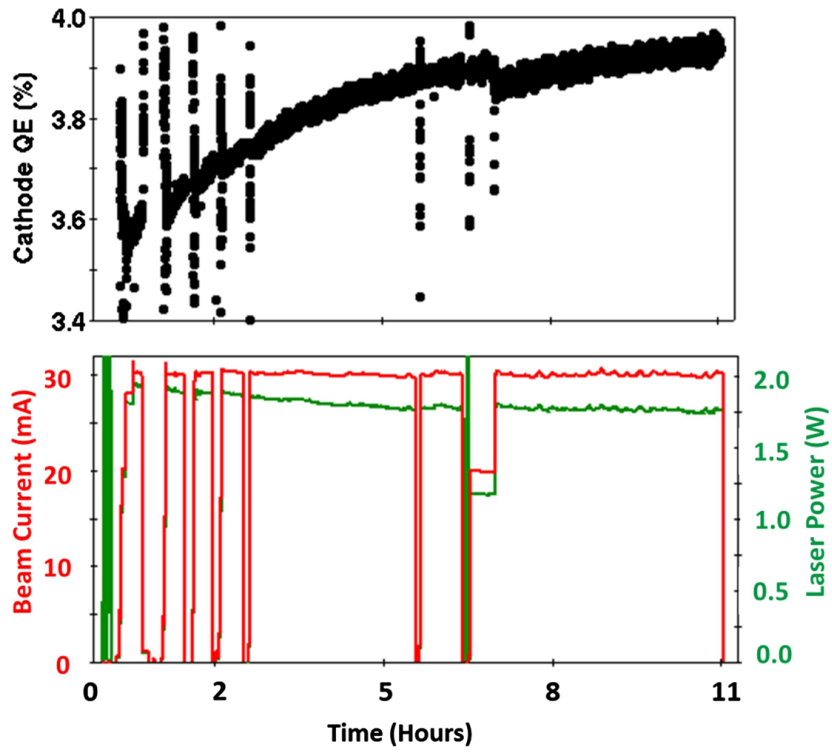

FIG. 10. High-current $9 \mathrm{MHz} \mathrm{CW}$ operation (up to $30 \mathrm{~mA}$ ) with laser beam intensity feedback. Top: Cathode QE (black). Bottom: Average laser power delivered to the cathode (green) and beam current measured by the DCCT (red).

\section{COOLING DEMONSTRATION}

During the spring of 2019, a new rf timing system, allowing for the $76 \mathrm{kHz}$ mode of operation, was commissioned. The $76 \mathrm{kHz}$ frequency corresponds to the RHIC revolution frequency at a gamma of 4.1. Cooling commissioning started with the $76 \mathrm{kHz}$ mode of operation. In this mode, the average electron beam current and power are reduced by 2 orders of magnitude in comparison to the $9 \mathrm{MHz} \mathrm{CW}$ mode. At the same time, the beam quality in the $76 \mathrm{kHz}$ mode is unchanged from the pulsed mode.

Using more than one macrobunch per train generates significant energy differences between macrobunches (see Fig. 5) As a result, the energy of only one electron

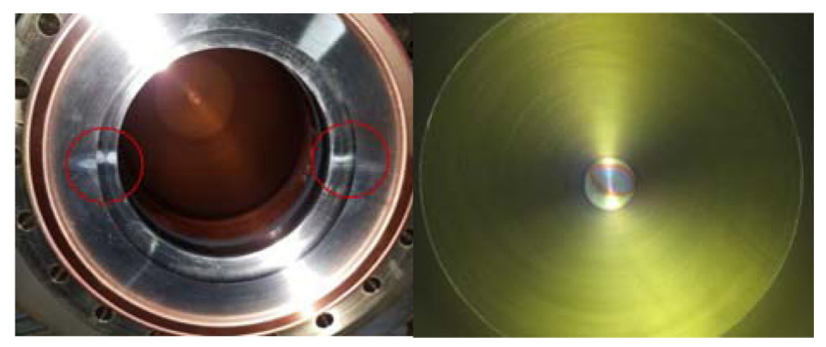

FIG. 11. Marks on the high-power beam dump surface as a result of a high-power beam operation with $20 \mathrm{~mA}$ beam current and 1.6 MeV kinetic energy during the 2018 run. Left: Beam dump entrance flange with marks at both sides highlighted by red circles. Right: Enlargement of the end of the cone. 

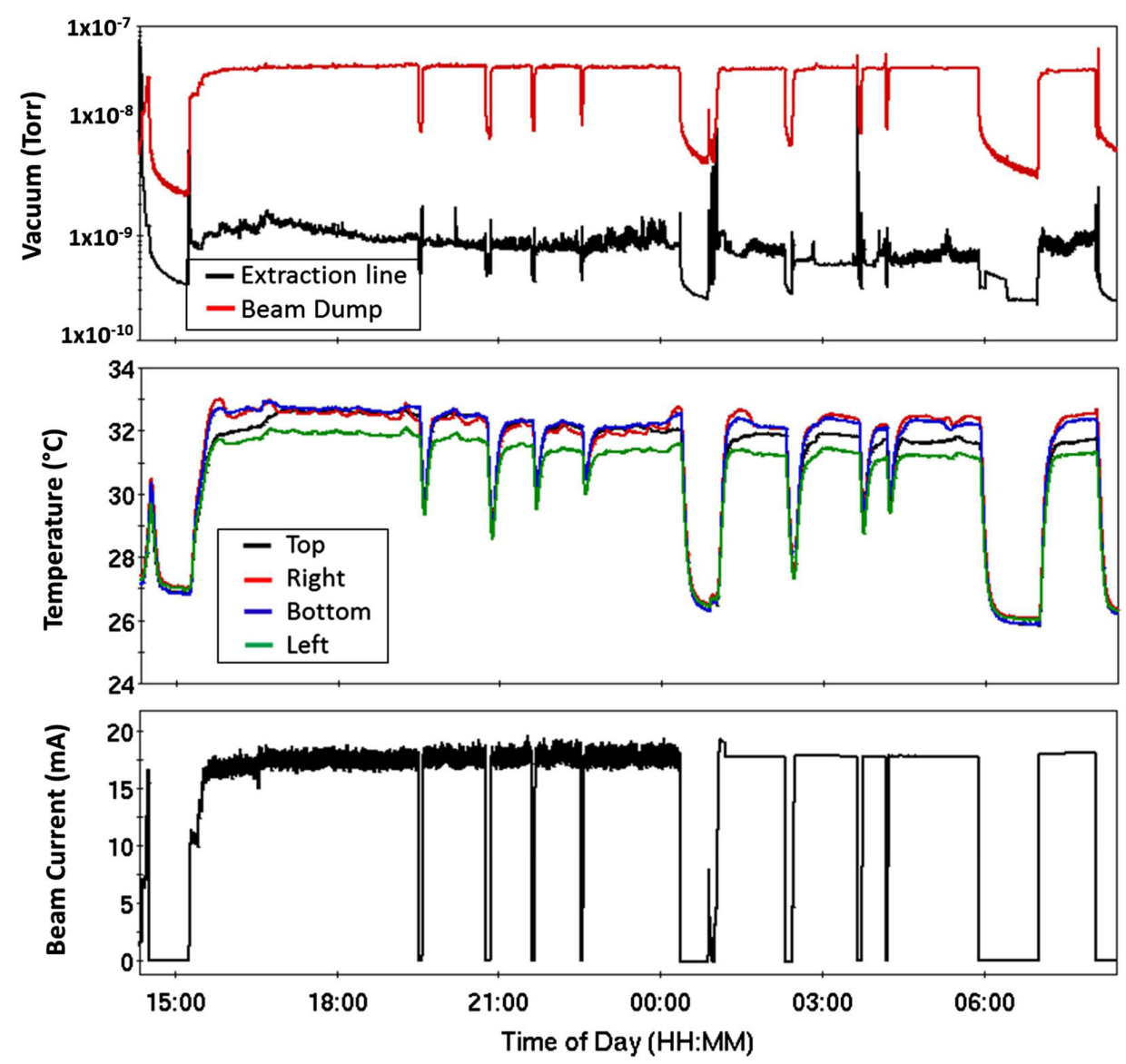

FIG. 12. High-current $9 \mathrm{MHz} \mathrm{CW}$ operation to the high-power beam dump at the end of run 2019 with an average beam current of up to $18 \mathrm{~mA}$ and $2 \mathrm{MeV}$ kinetic energy. Top: Vacuum in the extraction line and near the beam dump. Middle: Temperatures of the different sides of the beam dump entrance pipe. Bottom: Beam current measured by the DCCT. Intensity feedback was turned on at 01:00 AM, and the fast intensity fluctuations were reduced from $5 \times 10^{-2}$ to $5 \times 10^{-4}$ peak to peak.

macrobunch can be set to provide cooling. Therefore, the $76 \mathrm{kHz}$ beams can be used to cool a single ion bunch in each of the RHIC rings.

For the initial cooling commissioning, the lowest BES-II RHIC gold ions energy of $3.85 \mathrm{GeV}$ per nucleon was used. The respective ion beam parameters are listed in Table II.

In April 2019, the first cooling of a single ion bunch using bunched electron beam was demonstrated [30].

TABLE II. Ion beam parameters used during electron cooling commissioning at RHIC.

\begin{tabular}{lcc}
\hline \hline Parameter & Unit & Value \\
\hline Species & $\mathrm{GeV} /$ nucleon & $\mathrm{Au}$ \\
Energy & $10^{8}$ & $65,4.59$ \\
Intensity per bunch & $10^{-4}$ & $4-5$ \\
Energy spread & $\mu \mathrm{m}$ & 1.5 \\
Normalized emittance & $\mathrm{kHz}$ & 76 \\
Revolution frequency & $\mathrm{MHz}$ & 9.1 \\
rf frequency & & 111 \\
Numbers of bunches per ring & &
\end{tabular}

After successful commissioning of the cooling in one ring, we demonstrated that the same electron beam can be used to cool ions in the second RHIC ring using the $76 \mathrm{kHz}$ mode. Eventually, cooling in both RHIC rings in the $9 \mathrm{MHz}$ $\mathrm{CW}$ mode was demonstrated. This allowed us to achieve cooling of the colliding ion bunches and to increase the integrated luminosity of RHIC by $40 \%$ over the length of the store.

One of our initial concerns was that the electron beam quality in the $9 \mathrm{MHz} \mathrm{CW}$ high-current mode could be different from the beam quality in the pulsed mode. For example, the beam quality could degrade due to the ion accumulation in the transport line or due to the changed response of the rf system in the $\mathrm{CW}$ mode. To measure the beam quality at high-current operation, alternative beam diagnostics were considered: a beam-induced gas fluorescent profile monitor, a wire scanner, and a boron nitride nanotube screen [31]. Unfortunately, none of these diagnostics were ready to use during the LEReC beam commissioning. Instead, to confirm that the electron bunch parameters in the $\mathrm{CW}$ mode did not change, we used the electron cooling efficiency as the beam quality indicator. 


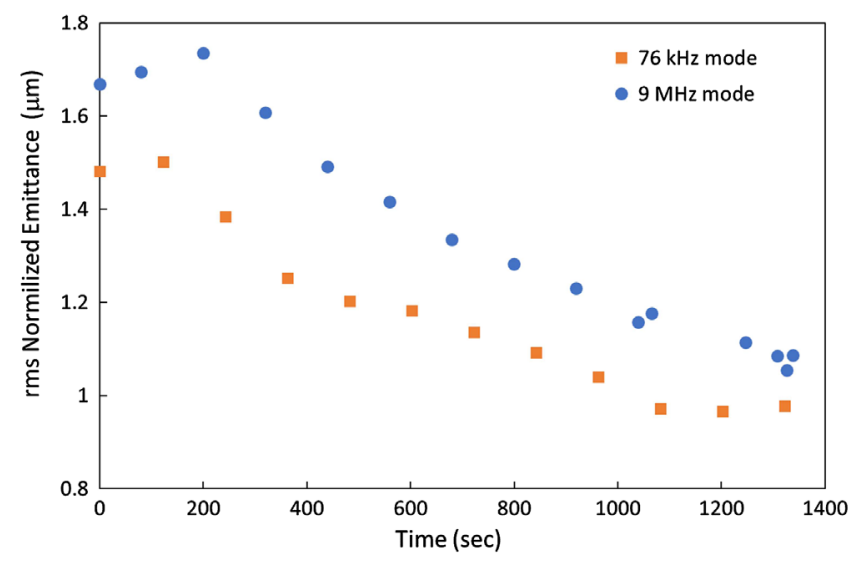

FIG. 13. Emittance reduction of a single RHIC ion bunch in the RHIC yellow ring as a result of LEReC providing cooling in the $76 \mathrm{kHz}$ (orange) and in the $9 \mathrm{MHz}$ (blue) modes. Cooling started around the 200 s mark.

We confirmed that the ion cooling rate was comparable in the $76 \mathrm{kHz}$ mode and in the $9 \mathrm{MHz} \mathrm{CW}$ mode, as shown in Fig. 13.

At the end of the run, electron cooling at another gold beam energy of $4.6 \mathrm{GeV}$ per nucleon was commissioned using electron beams with an energy of $2 \mathrm{MeV}$. For example, cooling of six ion bunches in both RHIC rings simultaneously using a $2 \mathrm{MeV}$ electron beam is shown Fig. 14 during the last day of RHIC operation at an energy of $4.6 \mathrm{GeV}$ per nucleon. First, it shows the evolution of the bunch length during a regular store without cooling, then during RHIC store with cooling.

\section{SUMMARY}

A state-of-the-art electron accelerator, which provides a beam suitable for electron cooling at Lorentz $\gamma$ factors of 4.1 and 4.9, was designed, built, and commissioned. Unlike previous dc electron coolers, LEReC uses if accelerated electron bunches that cool ions simultaneously in the two rings of the collider. A particular challenge with rf accelerated beams is to maintain the small momentum spread of the electron bunches, while also meeting the requirement for low transverse emittance and high current.

In this paper, we described our experience with the commissioning of the world's first rf-based electron cooler and with the attainment of an electron beam of the required quality [32].
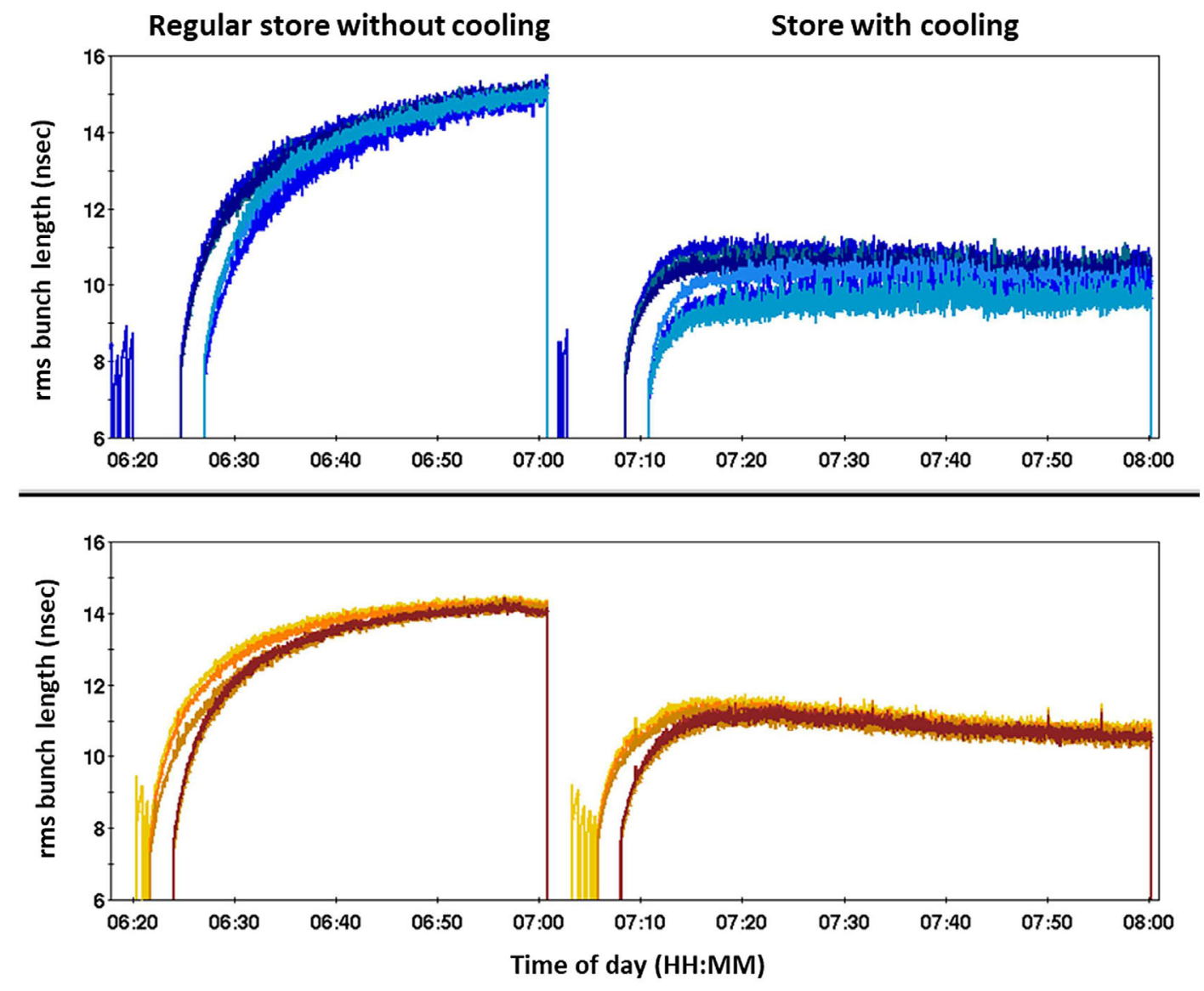

FIG. 14. Bunch length reduction in both RHIC rings as a result of longitudinal electron cooling running in the $9 \mathrm{MHz} \mathrm{CW}$ mode. Top: Bunch length of the first three and the last three bunches in the blue ring. Bottom: Bunch length of the first three and the last three bunches in the yellow ring. 


\section{ACKNOWLEDGMENTS}

The work was supported by many people from various groups of the Collider-Accelerator and other Departments of Brookhaven National Laboratory as well as Cornell University, Fermi National Accelerator Laboratory, Argonne National Laboratory, and Jefferson Lab. The authors are grateful to V. Litvinenko and T. Roser for help and useful suggestions during different stages of LEReC commissioning. This work was supported by Brookhaven Science Associates, LLC, under Contract No. DESC0012704 with the U.S. Department of Energy.

[1] D. Kayran et al., First results from commissioning of low energy RHIC electron cooler (LEReC), in Proceedings of the 10th International Particle Accelerator Conference (IPAC'19), Melbourne, Australia, 2019 (JACoW, Geneva, 2019), pp. 769-772.

[2] A. Fedotov, Bunched beam electron cooling for low energy RHIC operation, ICFA Beam Dynamics letter No. 65, 2014, p. 22.

[3] C. Liu et al., Improving the luminosity for beam energy scan II at RHIC, in Proceedings of the 10th International Particle Accelerator Conference (IPAC'19), Melbourne, Australia, 2019 (JACoW, Geneva, 2019), pp. 540-543.

[4] G. I. Budker, An effective method of damping particle oscillations in proton and antiproton storage rings, Sov. At. Energy 22, 438 (1967).

[5] H. Poth, Electron cooling: Theory, experiment, application, Phys. Rep. 196, 135 (1990).

[6] S. Nagaitsev et al., Experimental Demonstration of Relativistic Electron Cooling, Phys. Rev. Lett. 96, 044801 (2006).

[7] A. V. Fedotov et al., Accelerator physics design requirements and challenges of $\mathrm{rf}$ based electron cooler LEReC, in Proceedings of the North American Particle Accelerator Conference (NAPAC'16), Chicago, IL, 2016 (JACoW, Geneva, 2016), pp. 867-869.

[8] B. Dunham et al., Record high-average current from a high-brightness photoinjector, Appl. Phys. Lett. 102, 034105 (2013).

[9] D. Kayran et al., First results of commissioning dc photogun for RHIC low energy electron cooler (LEReC), in Proceedings of the 59th Advanced ICFA Beam Dynamics Workshop on Energy Recovery Linacs (ERL'17), Geneva, 2017 (JACoW, Geneva, 2017), pp. 65-69.

[10] E. Wang et al., Bi-alkali antimonide photocathodes for LEReC dc gun, in Proceedings of the 10th International Particle Accelerator Conference (IPAC'19), Melbourne, 2019 (JACoW, Geneva, 2019), pp. 2154-2156.

[11] Z. Zhao, B. Sheehy, and M. Minty, Generation of $180 \mathrm{~W}$ average Green power from fiber laser, Opt. Express 25, 8138 (2017).

[12] L. K. Nguyen et al., Active pointing stabilization techniques applied to the low energy RHIC electron cooling laser transport at $\mathrm{BNL}$, in Proceedings of the North American Particle Accelerator Conference (NAPAC'19), Lansing, MI, 2019, paper THYBA6.
[13] P. Inacker et al., Design of the 2-stage laser transport for the low energy RHIC electron cooling dc photogun, in Proceedings of the North American Particle Accelerator Conference (NAPAC'19), Lansing, MI, 2019, paper MOPLM18.

[14] C. J. Liaw et al., Cathode puck insertion system design for the LEReC photoemission dc electron gun, in Proceedings of the North American Particle Accelerator Conference (NAPAC'16), Chicago, IL, 2016 (JACoW, Geneva, 2016), pp. 1021-1023.

[15] W. Xu et al., Commissioning of the SRF booster cavity for LEReC, in Proceedings of the 29th Linear Accelerator Conference (LINAC2018), Beijing, 2018 (JACoW, Geneva, 2018), pp. 40-43.

[16] B. P. Xiao et al., Higher order mode damper for low energy RHIC electron cooler superconducting radio frequency booster cavity, Phys. Rev. Accel. Beams 22, 050101 (2019).

[17] B.P. Xiao et al., HOM consideration of $704 \mathrm{MHz}$ and 2.1 GHz cavities for LEReC linac, in Proceedings of the 7th International Particle Accelerator Conference (IPAC'16), Busan, Korea, 2016 (JACoW, Geneva, 2016), pp. 528-531.

[18] B. P. Xiao et al., Design and test of $704 \mathrm{MHz}$ and $2.1 \mathrm{GHz}$ normal conducting cavities for low energy RHIC electron cooler, Phys. Rev. Accel. Beams 22, 030101 (2019).

[19] T. Xin et al., Measuring the electrical center and field flatness of $704 \mathrm{MHz}$ deflecting cavity for LEReC with wire stretching system, in Proceedings of the 9th International Particle Accelerator Conference (IPAC'18), Vancouver, 2018 (JACoW, Geneva, 2018), pp. 1320-1322.

[20] S. Polizzo, 9 MHz LEReC cavity design, in Proceedings of the Eighth Continuous Wave and High Average Power RF Workshop, Trieste, Italy, 2014, https://indico.cern.ch/ event/276274/contributions/625681/attachments/501434/ 692494/LeRHIC_9MHz_Cavity_Presentation_Slides.pdf.

[21] J. Kewisch, A. V. Fedotov, D. Kayran, and S. Seletskiy, Beam optics for the RHIC low energy electron cooler (LEReC), in Proceedings of the North American Particle Accelerator Conference (NAPAC'16), Chicago, 2016 (JACoW, Geneva, 2016), pp. 1015-1017.

[22] Z. Zhao, K. Mernick, M. Costanzo, and M. Minty, An ultrafast laser pulse picker technique for high-averagecurrent high-brightness photoinjectors, Nucl. Instrum. Methods Phys. Res., Sect. A 959, 163586 (2020).

[23] A. Miller et al., LEReC instrumentation design and construction, in Proceedings of the 5th International Beam Instrumentation Conference (IBIC'16), Barcelona, 2016 (JACoW, Geneva, 2016), pp. 417-421.

[24] S. Seletskiy et al., Conceptual design of LEReC fast machine protection system, in Proceedings of the 5th International Beam Instrumentation Conference (IBIC'16), Barcelona, 2016 (JACoW, Geneva, 2016), pp. 665-668.

[25] X. Gu et al., Stable operation of a high-voltage highcurrent dc photoemission gun for the bunched beam electron cooler in RHIC, Phys. Rev. Accel. Beams 23, 013401 (2020).

[26] S. Seletskiy et al., Accurate setting of electron energy for demonstration of first hadron beam cooling with rfaccelerated electron bunches, Phys. Rev. Accel. Beams 22, 111004 (2019). 
[27] C. Liu et al., Transverse beam emittance measurements with multi-slit and moving-slit devices for LEReC, in Proceedings of the 7th International Beam Instrumentation Conference (IBIC'18), Shanghai, 2018 (JACoW, Geneva, 2018), pp. 486-489.

[28] M. Zhang, Emittance formula for slits and pepperpot measurement, Report No. Fermilab-TM-1988, 1996.

[29] R. R. Mammei et al., Charge lifetime measurements at high average current using a $\mathrm{K} 2 \mathrm{CsSb}$ photocathode inside a dc high voltage photogun, Phys. Rev. Accel. Beams 16, 033401 (2013).
[30] A. Fedotov et al., First electron cooling of hadron beams using a bunched electron beam, in Proceedings of the North American Particle Accelerator Conference (NAPAC'19), Lansing, MI, 2019, paper THZBA5.

[31] T. Miller et al., Overview of the beam instrumentation and commissioning results from the BNL low energy RHIC electron cooling facility, in Proceedings of the 8th International Beam Instrumentation Conference (IBIC'19), Malmo, Sweden, 2019, paper ID MOBO01.

[32] A. V. Fedotov et al., Experimental demonstration of hadron beam cooling using radio-frequency accelerated electron bunches [Phys. Rev. Lett. (to be published)]. 